\title{
NURSES' SENSE OF COHERENCE AND THEIR WORK RELATED PATTERNS OF BEHAVIOUR
}

\author{
MALGORZATA A. BASIŃSKA ${ }^{1}$, ANNA ANDRUSZKIEWICZ², and MAGDALENA GRABOWSKA ${ }^{1}$ \\ ${ }^{1}$ Kazimierz Wielki University in Bydgoszcz, Bydgoszcz, Poland \\ Institute of Psychology \\ ${ }^{2}$ Nicolaus Copernicus University in Torun, Toruń, Poland \\ Collegium Medicum
}

\begin{abstract}
Objectives: This paper is a report on the relationship between a sense of coherence (and its components) and work related patterns of behaviour in the group of nurses. Materials and Method: Data were collected by two questionnaires (Antonovsky's Orientation Questionnaire and Work Related Patterns of Behaviour and Experience Questionnaire) in the sample of 331 volunteer nurses employed in three Polish general care hospitals. Results: The analysis of r-Pearson's correlation and the linear multiple regression analysis revealed a strong relationship between the sense of coherence and healthy type of behaviour, and a strong negative relationship with a burnout type of behaviour. Conclusion: The results obtained in this study provide grounds for the conclusion that the sense of coherence correlates with healthy functioning at work in the group of nurses.
\end{abstract}

Key words:

Burnout, Health promotion, Nurses, Occupational health, Stress

\section{INTRODUCTION}

Sense of coherence (SOC) is regarded as an important source of resilience and protection of an individual's health [1]. Individuals with a high level of sense of coherence enjoy better health and well-being [2,3] and, owing to a different perception and event assessment, they experience a lower feeling of burden and they better cope with difficulties than individuals with a weak sense of coherence [4]. They also more frequently take up important problems and accomplish tasks assigned to them [5]. A strong sense of coherence is an incentive to activity. In the course of the activity, resources, cognition schemes and competences are used more effectively, and the detrimental effect of stressors can be reduced or reversed. The research problem put forward in this study concerns the relationship between the sense of coherence and its components on the one hand and the pattern of work behaviour in a group of nurses on the other. Presumably, taking into account both the theoretical assumptions and study reports, the sense of coherence can play an important role in the nurses' struggle against professional burden.

The following research questions have been put forward:

1. Is there a connection between the sense of coherence (and its components) and nurses' work related patterns of behaviour?

2. Is the sense of coherence (and its components) a predictor of nurses' work related patterns of behaviour?

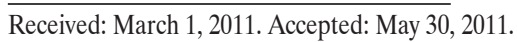

Address reprint request to M. Grabowska, Kazimierz Wielki University in Bydgoszcz, Staffa 1, 85-867 Bydgoszcz, Poland (e-mail: magda-gra@wp.pl). 


\section{Literature review: the context of the study}

\section{Sense of coherence}

According to Aaron Antonovsky [1, p. 11], a sense of coherence is "a general orientation which expresses the degree to which an individual feels an intense, yet dynamic, feeling of predictability of the internal and external environment and also that situations will, most probably, turn out to be as favourable as expected, based on rational assumptions". It consists of three components: comprehensibility, manageability and meaningfulness. The sense of comprehensibility is a degree to which an individual perceives the external and internal stimuli as cognitively sensible and coherent and such that can be coped with. Antonovsky [1] understood manageability as the extent to which an individual perceived the available resources as sufficient to satisfy his/her needs. He also claimed that meaningfulness was the extent to which an individual felt the sense of life from the emotional perspective. It expresses the motivation side and is the most important component of the sense of coherence. Despite valuating its various components, Antonovsky [1] strongly emphasised the indivisibility of the sense of coherence.

Being a relatively constant and stable disposition $[1,3]$ to perceive the world as understandable, controllable and reasonable, the sense of coherence is related to resistance to stress and helps understand individual differences in responding to difficult situations [6]. Numerous studies have positively verified Antonovsky's proposition concerning the buffer role of the sense of coherence in stress situation [6-9]. Individuals with a stronger sense of coherence more easily accept inevitable difficulties [10], are in a better physical and mental state [11,12], they are generally more satisfied with life $[13,14]$ and value it more. They are in better control of themselves and situations which they are in, and experience less anxiety [15] and hostility [16]. The sense of coherence is regarded as an important variable related to the professional functioning of an individual. SOC can reduce the perception of burden related to one's job $[4,17]$.
Therefore, it seems that the sense of coherence is more important in terms of maintaining one's health than other adaptational factors [18].

It can be claimed that the positive effect of the sense of coherence also covers the professional life of an individual. Study results seem to indicate that, being a relatively stable disposition, SOC affects the ability to mobilise and generate the social resources of an individual at workplace [3], and when strong, it also protects one against the negative impact of being unemployed [19]. The research conducted on Swedish social workers has shown that the lower was their sense of coherence, the more frequently they experienced psychosomatic abnormalities. These adverse conditions at work resulted in emotional exhaustion and depersonalisation [20]. A study involving Polish social workers clearly indicated the existence of a positive correlation between the sense of coherence and healthy patterns of work related behaviour and a negative correlation between SOC and the burnout type of behaviour at work [21].

A Finnish study on 477 nurse educators has revealed a positive correlation between the sense of coherence and professional functioning. The higher the sense of coherence, the more satisfying one's job, the less intensely perceived the job burden and negative effects of stress, the higher self-assessment of one's own professional competence and the higher assessment of one's health status [22].

A study of Polish nurses showed that when their global sense of coherence was strong, they behaved according to the healthy type $\mathrm{G}$ or the frugal type $\mathrm{S}$ at work more frequently, and they behaved according to the burnout type B less frequently than the nurses with a weak sense of coherence [23]. The stronger the nurses perceive reality as understandable and ordered (understandability), the stronger their conviction that they can change it and influence it (sense of control) and the stronger their feeling that it is worth doing (meaningfulness), the weaker their feeling of burnout [24]. Summing up the international research results, it can be claimed that a strong sense of coherence is a manifestation 
of healthy functioning of an individual, including functioning at work, which occupies a special position among various human activities. Job satisfaction originates from the fact that work satisfies various needs related to certain expectations; also it helps maintain and develop one's selfesteem. Antagonistically to satisfaction of job, functioning in a profession can be a source of dissatisfaction [25].

\section{Professional burnout}

The problem of occupational burnout is becoming an increasingly common phenomenon, concerning more and more professions. It is usually defined, after Maslach, as a syndrome of emotional exhaustion, depersonalisation and decreased sense of self-achievement, which can occur in individuals who work with other people in a certain manner [26]. Conditions and mechanisms of burnout are not clear as the factors that favour burnout and which protect one from it interact. On the one hand, these are the characteristics which are specific to an individual, and on the other - positive or negative characteristics of one's professional environment [26]. And although the research on Polish police officers [27] showed a positive correlation between the stress experienced at work and the intensity of occupational burnout, neither the stress itself nor the specificity of the work environment sufficiently explain the phenomenon of occupational burnout [26]. This means that the specific character of an individual's traits and properties could be an important factor affecting the burnout process.

Occupational burnout affects a considerable proportion of nurses; e.g., two thirds of the studied population in the UK complained of professional exhaustion [28], which resulted in higher absenteeism [29].

From the existential perspective, the phenomenon of burnout may also be perceived as a consequence of lacking the meaning of work [30]. This theoretical perspective is strongly connected with the sense of coherence and its component - meaningfulness.

\section{Work related behaviour and experience}

It is commonly known that not everyone experiences occupational burnout syndrome. This depends largely on the individual's attitude to professional requirements [26]. Consequently, researchers started to ponder whether, in the face of increasing professional requirements, there are any types of behaviour at a workplace with a positive and negative effect on health. Trying to find the answer to this problem, Schaarschmidt and Fischer [31] developed a model of work related behaviours and experiences and a tool to measure them. The authors based their model on an interactive approach, trying to determine the resources being at the individual's disposal in the context of coping with various occupational situations [25]. They determine the experiences and behaviour of individuals in three spheres of their functioning. Within each of them they describe the components which make them up. These are [31]:

1. Professional commitment, which consists of: subjective importance of work; professional ambitions; readiness to commit oneself to professional tasks; striving for perfection; ability to maintain distance and to relax after work.

2. Resistance to stress that consists of: ability to come to terms with failure; optimistic attitude to professional challenges and demands; inner calm and balance.

3. Emotional well-being, which consists of: a feeling of professional success; satisfaction with life in general; a sense of social support from one's closest circle [25,32].

Cluster analysis of the data in the reference sample led to four different types of work-related experience and behaviour patterns.

Type $\mathrm{G}$ - the healthy type

The main determinant of the $G$ type is a configuration of professional commitment dimensions: high professional ambitions in combination with a moderate subjective 
importance of one's work and a moderate tendency to output energy. Mental resilience is expressed by a tendency to keep one's distance, rather low tendency to give up in the face of failure, a tendency to solve problems in an active manner and the feeling of inner mental comfort. A high significance of the feeling of professional accomplishment, satisfaction with life and social support are complements of the healthy G type.

\section{Type $S$ - the frugal type}

Type $\mathrm{S}$ individuals are characterised by a subjectively low significance of work, low level of professional ambitions, low level of readiness to output energy and absence of striving for perfection when performing tasks. The major characteristic distinguishing this type from other patterns of behaviour is a tendency to keep one's distance to work related problems. A relatively low tendency to resign in combination with a low commitment indicates that the frugal type of behaviour is not a result of resignation. Such individuals have a positive attitude to their professional role, which is reflected in their satisfaction with life and a high significance of inner balance. They have a moderate sense of accomplishment and their optimistic attitude to life may indicate that they derive satisfaction from situations outside their profession. Both the $\mathrm{G}$ and $\mathrm{S}$ types reflect a positive and healthy attitude to work.

\section{Type A — risk type (overburdened)}

The characteristic features of this type include high professional commitment and attaching high significance to work as well as a perfectionist attitude to professional tasks. What is most dangerous is a low ability to distance oneself from one's professional duties. A-type individuals find it difficult to relax in their leisure time. They have weak mental resilience, feelings of mental discomfort and rather high tendency to resign in the face of failure. Diligence, scrupulosity and perfectionism are the primary traits of the personality of A-type individuals.
Dissatisfaction with life and lack of social support from the people bring about negative emotions. The discrepancy between the efforts made to perform one's task and the expected remuneration can cause so called remuneration crisis, which may result in an increased risk of psychosomatic diseases.

Type B - the burnout type

This type is characterised by very weak significance of work, weak resistance to stress along with a very weak ability to distance oneself from job, readiness to resign in the face of a difficult situation and, finally, by the lack of harmony and inner balance. There is a similarity between the $\mathrm{B}$ and the $\mathrm{S}$ types in their weak significance of work; however, unlike the frugal type, the burnout type has a low ability to distance oneself. This inability is a cause of work related burden and constant anxiety. Decreased motivation, decreased mental resilience and predominance of negative emotions in B-type individuals become predictors of the syndrome of professional burnout. The feeling of professional burnout emerges slowly in the form of mental fatigue. With its growth, a sense of helplessness appears, not only in the professional sphere, but also in one's private life. The last phase of burnout can be accompanied by the feeling of pointlessness of life, which is a serious threat to an individual's mental and physical health $[25,31,32]$.

The latter two types are worth special attention. The patterns of experiences and behaviour associated with work, which are shown here, pose a serious threat to individual's mental health, thereby hampering the person's development.

The main aim of the study was to assess the relationship between the sense of coherence and its components in a group of nurses on the one hand and their patterns of behaviour in the workplace on the other. It is also very important that this research project - thanks to Work Related Patterns of Behaviour and Experience 
Questionnaire - allows describing precisely the work related patterns of behaviour - from healthy one to burnout.

Based on the theoretical analysis of the problem and our previous results, it has been assumed that a higher sense of coherence is associated with a healthy type of behaviour at work and it protects one against the occupational burnout. On the other hand, a weak sense of coherence correlates positively with the burnout type of work behaviour $[21,23]$. Focusing on the study of the above dependencies, it is important to be aware that other factors (e.g. political, organizational and situational) also perform a vital role in functioning at work, but they are not the subjects of this work.

\section{MATERIALS AND METHOD}

\section{Design}

This study was of quantitative character and a model of correlation was used. The participating nurses completed two questionnaires: Antonovsky's Orientation Questionnaire - SOC-29 and Work-Related Behaviour and Experience Pattern - AVEM. The tests were collected at an arranged time.

All nurses working in huge, multi-ward hospitals in a town of about 400000 inhabitants were invited, during a joint meeting, to take part in the survey, but not all took advantage of this opportunity. Those who did participate were volunteers. After stating their consent, the participants received the questionnaire sheets and completed them individually.

\section{Ethical considerations}

We received ethical committee's approval to conduct this research project. The study was anonymous and the results were analyzed in groups. The nurses participating were not related in any way to anyone implementing the study. Returning completed questionnaire sheets by the nurses was deemed to be a sign of their consent to participate in the research.

\section{Sample}

The group under study consisted of 331 female nurses employed in various hospital wards. All of them worked shifts. The mean age was 34.15 (SD $=6.61$ ), with the youngest one aged 21, and the oldest 52. Most of them were married $(\mathrm{n}=214 ; 65 \%), 87$ of them were single (non-married) (26\%) and rest of them were divorced $(\mathrm{n}=30 ; 9 \%)$. The nurses were graduates either of secondary nursing schools, or of medical university, but in Poland these two groups of nurses are equal in their professional status. Nowadays in Poland, in accordance with a new law, all secondary nursing schools are closed down and the only way to become a nurse is to graduate from a medical university.

\section{Measurements and data analysis}

The data were collected during the years 2006 and 2007 using the following questionnaires. The sense of coherence was examined with the use of Antonovsky's Life Orientation Questionnaire - SOC-29 (1995), which measures global sense of coherence and its components (comprehensibility, manageability and meaningfulness). SOC-29 consists of 29 items, each has a 7-point scale estimate with described extremes ( 7 - maximum, 1 - minimum). The questionnaire included three subscales corresponding to the components of the sense of coherence: comprehensibility (11 statements), manageability (10 statements) and meaningfulness ( 8 statements). Global SOC is the sum of these three components [1].

The types of work related behaviour were examined with the use of Schaarschmidt's and Fischer's Work Related Patterns of Behaviour and Experience Questionnaire AVEM [25], which describes four types of work related behaviour: type $\mathrm{G}$ - healthy, type $\mathrm{S}$ - frugal, type A — risk (overburdened), type B - burnout. AVEM Questionnaire 
consists of 66 items, each has a 5-point scale estimate where 1 means "I strongly agree", and 5 means "I strongly disagree". The results are calculated by a computer programme, which calculates the values for work related patterns of behaviour: G, S, A and B, which, as a whole, add up to one. The higher is the fraction, the stronger is the tendency to function according to a given type of behaviour [25].

The assessment of the Polish version of the SOC-29 Questionnaire showed very high reliability. The internal compliance indicators were for the sense of coherence -0.92 , sense of comprehensibility -0.78 , sense of manageability -0.72 and the sense of meaningfulness -0.68 , whereas Cronbach's alpha $=0.78$ [24].

AVEM is a highly reliable tool. For the scales of the Polish version, the internal reliability - Cronbach's alpha ranged from 0.71 to 0.84 [25].

The resultant data were analysed using the statistical package Statistica 6.0. All of the research variables are quantitative ones. The Shapiro-Wilk test was used to estimate the distribution of the research variables.

The mean results and standard deviation of the test group of nurses were calculated. The association between global sense of coherence and its components (comprehensibility, manageability and meaningfulness) and four types of behaviour was tested at first using Pearson's correlation test. Next, the linear multiple regression analysis was used in order to determine the extent to which the sense of coherence and its components explained the behaviour of individuals in the workplace. Both Pearson's correlation test and regression analysis made it possible to answer the questions: (1) Is there a correlation between research variables; (2) What is the direction of the relationship mentioned above, its strength and participation of independent variables in explaining the dependent ones? Answering these questions was essential in the process of answering the research questions listed above.

\section{RESULTS}

\section{Descriptive results}

The sense of coherence in the study group varied. The following results were obtained: manageability from 20 to 71 , with an acceptable range from 10 to $70(\mathrm{M}=44.69$; $\mathrm{SD}=9.15)$; comprehensibility from 16 to 56 , with an acceptable range from 11 to $77(\mathrm{M}=43.43 ; \mathrm{SD}=6.82)$; meaningfulness from 24 to 68 with an acceptable range from 8 to $56(\mathrm{M}=48.30$; $\mathrm{SD}=8.99)$; global sense of coherence from 76 to 192, with an acceptable range from 29 to 203 ( $\mathrm{M}=136.46 ; \mathrm{SD}=21.43)$.

The nurses function at work in various manners; almost all the possible patterns of behaviour can be observed; however, a majority of subjects behave according to type A - overburdened and type B - burnout. About a quarter of the nurses in the study group behave according to the healthy pattern - G, while the lowest number - according to the frugal type $-\mathrm{S}$ (Table 1$)$.

Table 1. Descriptive statistics obtained by the nurses $(\mathrm{N}=331)$ in the scales of the AVEM questionnaire

\begin{tabular}{lcc}
\hline Pattern of behaviour & Mean & Standard deviation \\
\hline Healthy type-G & 0.25 & 0.329 \\
Frugal type-S & 0.16 & 0.259 \\
Overburdened type-A & 0.31 & 0.328 \\
Burnout type-B & 0.28 & 0.344 \\
\hline
\end{tabular}

\section{The relationship between the sense of coherence and work related patterns of behaviour}

The next phase of the statistical analysis was to search for a relationship between the intensity of global sense of coherence and its components on the one hand, and probability of functioning in workplace according to a given type of behaviour on the other (Table 2 and 3). R-Spearman's test was applied because the distribution was not normal. A statistically significant positive correlation was observed between the global sense of coherence and its components 
Table 2. R-Spearman's correlations between global sense of coherence and its components on the one hand and work related patterns of behaviour in a group of nurses $(\mathrm{N}=331)$ on the other

\begin{tabular}{lcccc}
\hline \multicolumn{1}{c}{ Pattern of behaviour } & Manageability & Comprehensibility & Meaningfulness & SOC \\
\hline Healthy type-G & $0.34^{1}$ & $0.48^{1}$ & $0.46^{1}$ & $0.50^{1}$ \\
Frugal type-S & $0.16^{2}$ & $0.12^{3}$ & $0.21^{1}$ & $0.20^{1}$ \\
Overburdened type-A & -0.09 & -0.06 & $-0.15^{2}$ & $-0.13^{3}$ \\
Burnout type-B & $-0.39^{1}$ & $-0.52^{1}$ & $-0.55^{1}$ & $-0.57^{1}$ \\
\hline
\end{tabular}

Level of significance: ${ }^{1} \mathrm{p}<0.001,{ }^{2} \mathrm{p}<0.01,{ }^{3} \mathrm{p}<0.05$.

on the one hand and the healthy type $-\mathrm{G}$ and the frugal type $-\mathrm{S}$ of work related behaviour on the other. A statistically significant negative correlation was observed between the global sense of coherence along with its components and the burnout type - B and the overburdened type A of work related behaviour. A statistically significant negative correlation was observed between the feeling of sense and the burnout type - B and the overburdened type A of work related behaviour. The other variables manageability and comprehensibility - as well as the global sense of coherence did not show any relationship with the latter work related pattern of behaviour.

The sense of coherence and its components explain as much as $28 \%$ of the variability of burnout pattern of behaviour in nurses and $21 \%$ and $7 \%$ of healthy and frugal pattern of behaviour, respectively (Table 3 ).

Taken separately, most of the components of the sense of coherence explain the types of work related behaviours

Table 3. Standard multiple linear regression analysis for assessing the interaction between sense of coherence and its components and patterns of work related behaviour in a group of nurses $(\mathrm{N}=331)$

\begin{tabular}{lcccc}
\hline \multirow{2}{*}{ Pattern of behaviour } & \multicolumn{4}{c}{ Sense of coherence } \\
\cline { 2 - 5 } & $\mathrm{R}$ & $\mathrm{R}^{\wedge} 2$ & $\mathrm{~F}(4.326)$ & $\mathrm{p}$ \\
\hline Healthy type-G & 0.464 & 0.215 & 22.373 & 0.000 \\
Frugal type-S & 0.271 & 0.074 & 6.465 & 0.001 \\
Overburdened type-A & 0.130 & 0.017 & 1.407 & 0.327 \\
Burnout type-B & 0.530 & 0.281 & 31.901 & 0.000 \\
\hline
\end{tabular}

(Table 4), but the percentage of variation explained by these independent variables is low.

Table 4. The impact of nurses' sense of coherence (and its components) on work related behaviour patterns - results of standard multiple linear regression analysis

\begin{tabular}{lcccc}
\hline \multicolumn{1}{c}{ Variables } & $\beta$ & $\mathrm{R}^{\wedge} 2$ & $\mathrm{t}(329)$ & $\mathrm{p}$ \\
\hline Healthy type-G & & & & \\
manageability & 0.304 & 0.092 & 5.780 & 0.000 \\
comprehensibility & 0.432 & 0.187 & 8.698 & 0.000 \\
meaningfulness & 0.422 & 0.178 & 8.438 & 0.000 \\
SOC & 0.444 & 0.198 & 8.999 & 0.000 \\
Frugal type-S & & & & \\
manageability & 0.145 & 0.021 & 2.662 & 0.008 \\
comprehensibility & 0.165 & 0.027 & 3.040 & 0.003 \\
meaningfulness & 0.258 & 0.067 & 4.852 & 0.000 \\
SOC & 0.225 & 0.051 & 4.185 & 0.000 \\
Overburdened type-A & & & & \\
manageability & -0.076 & 0.006 & -1.386 & 0.167 \\
comprehensibility & -0.061 & 0.004 & -1.115 & 0.266 \\
meaningfulness & -0.119 & 0.014 & -2.182 & 0.030 \\
SOC & -0.103 & 0.011 & -1.881 & 0.061 \\
Burnout type-B & & & & \\
manageability & -0.336 & 0.113 & -6.467 & 0.000 \\
comprehensibility & -0.484 & 0.234 & -10.032 & 0.000 \\
meaningfulness & -0.495 & 0.245 & -10.331 & 0.000 \\
SOC & -0.506 & 0.256 & -10.632 & 0.000 \\
\hline
\end{tabular}




\section{DISCUSSION}

\section{Sense of coherence in the group of nurses}

The analysis of these results reveals the existence of a relationship between the sense of coherence and the preferred pattern of behaviour in work - thereby the first research question was answered positively. There is a relationship between stronger sense of coherence and healthy pattern of work related behaviour (type $G$ and type $S$ ), while weaker sense of coherence is related to the burnout type of behaviour. The type A - overburdened - is not connected with sense of coherence with the exception of its one component - meaningfulness. This relationship has only been found for meaningfulness, but not for the global sense of coherence and its remaining two components. In attempting to answer the next research question, it may be concluded that sense of coherence and its components seem to be predictors of work related behaviour patterns: to the highest degree in the case of burnout and healthy type, and to a lower degree in the cases of the remaining types of the behaviour. The results of this study are very similar to those obtained in the study with social workers as participants [21]. In both studies a relationship was found between the sense of coherence and the following types: healthy - G, frugal $-\mathrm{S}$ and burnout $-\mathrm{B}$. The sense of coherence explains a large part of the variability of burnout and healthy behaviour at work in the group of nurses, with only a small part of frugal behaviour and practically no overburdened behaviour. The weak relationship between the sense of coherence and the overburdened type is noteworthy. It could be proposed that those who work too much, find it difficult to perceive their activities to be meaningful.

For people with the healthy type of behaviour, their occupation is a source of satisfaction and well-being, and a sense of coherence is also responsible for life satisfaction [17]. It also positively affects mental health if a person who behaves according to pattern $\mathrm{G}$ is able to keep her distance to work related problems. In the face of a failure, the problem is solved instead of being considered to represent an obstacle that stands in the way to achieving one's goal, which is a source of inner balance and harmony. Functioning according to healthy type $-\mathrm{G}$ or frugal type $-\mathrm{S}$ is related to resistance to stress and it is probably the sense of coherence that largely contributes to the relationship [6], as individuals with a strong sense of coherence have been found to accept the inevitable hardships in their lives much more easily [10]. People who represent the healthy pattern of behaviour tend to pursue ambitious goals, while being able to assess their effort depending on the difficulty of the tasks.

The ability to diagnose the types of behaviour and experiences in the workplace is particularly important in the context of the specific character of nurses' job. This enables evaluation of individual behaviour at work and both the negative and positive consequences of their behaviours on their health.

A strong sense of coherence means that a nurse is able to muster adequate resources for a given professional situation, and make full use of them. It also helps to recognize emotions associated with work and to deal better with problems, and when they cannot be resolved, it helps to adapt. Various research results show that sense of coherence plays the role of a buffer in stress reactions [6-9]. This buffer allows accepting inevitable difficulties [10], achieving higher life satisfaction [13,14], better self-control as a result of experiencing less anxiety [15] and hostility [16]. Our results above also show that the sense of coherence is a good predictor of nurses' healthy functioning in a workplace, which protects them against burnout. It is especially important factor in the context of general work satisfaction that is strongly connected to nurses' salaries [33], which are not very satisfying in Poland.

Stress in a workplace may be reduced with personal resources [34,35], and sense of coherence, as an element of these resources, may help the nurses to act professionally in their difficult and not very satisfying work environment. Thus, it can be concluded that the assessment of the sense of coherence and the types of behaviours and experiences at work should be performed as an integral part of the 
diagnosis of professional nurses in the context of dealing with their professional problems. Sense of coherence, as an important construct describing human behaviour, may be borrowed from the theory of salutogenesis and implemented to theories of nursing in order to enrich and broaden them. The value of the construct might be especially useful for health promotion and, as an intrapersonal factor, it might contribute to shaping of the environment due to the impact on human behaviour [36].

\section{Study limitations}

The main limitation of our study was that the respondents were volunteers. It is well known that volunteers are specific people, having predetermined personality traits. Another limitation of the study was that the research was restricted to nurses from big cities and big hospitals. Nurses working outside of huge hospitals, in small cities and in small health care unites were not included in the study.

\section{CONCLUSION}

This study shows that sense of coherence correlates with healthy functioning owing to the fact that it favours healthy patterns of work related behaviour and prevents those patterns of work related behaviour which are potentially harmful. The sense of coherence is more important in maintaining good health status than other adaptational factors [18]. It protects one from professional burnout and therefore its level should be taken into account in candidates for the profession of social worker; employees should be helped in increasing its level through various trainings.

Sense of coherence can be considered as a part of the personal resources of nurses - resources that play an important role in the process of coping with professional problems [21]. When facing new challenges of contemporary nursing, special attention should be paid to personal resources of nurses. Thus, it seems reasonable to take measures to improve the sense of coherence both in the process of training and in professional advice through organization of training courses, including those on interpersonal and relaxation training, and on issues of dealing with stress at work. It also appears to be essential to shape the skills of effective communication, empathy, improving the skills of using positive professional experiences and the development of emotional and professional support for nurses.

\section{Implications for practice and/or policy}

- The ability to diagnose the types of behaviour and experiences in the workplace is particularly important in the context of the specificities of nurses.

- The Work Related Patterns of Behaviour and Experience Questionnaire is a very good tool for diagnosis of burnout and other types of behaviour in the workplace.

- The assessment of the sense of coherence and the types of behaviours at work should be executed as an integral part of the diagnosis of nurses in the context of dealing with professional problems.

\section{CONTRIBUTORS \& ACKNOWLEDGEMENTS}

Substantial contributions to conception and design - MAB, AA, acquisition of data $-\mathrm{AA}$, or analysis and interpretation of data - MAB, AA. Drafting the article - MAB or revising it critically for important intellectual content - AA, MG. Final approval of the version to be published - MAB, MG.

\section{REFERENCES}

1. Antonovsky A. Unravelling the mystery of health. How people manage stress and stay well. Warszawa: Fundacja IPN; 1995 [in Polish].

2. Basińska M, Kossakowska M, Liczmańska J. The divergence between real-self and ideal-self and a sense of coherence among people with multiple sclerosis. In: Aouil B, editor. Handicap Man. Risk and Development Chance. Bydgoszcz: WSP; 1999. p. 236-49 [in Polish]. 
3. Feldt T, Kivimaki M, Rantala A, Tolvanen A. Sense of coherence and work characteristics: a cross-lagged structural equation model among managers. J Occup Organ Psychol 2004;77:323-42.

4. Coe RM, Miller DK, Flaherty JH. Sense of coherence and perception of caregiving burden. Behav Health Aging 1991;2(2):93-9.

5. Yeheskel A. The intimate environment and the sense of coherence among Holocaust survivors. Soc Work Health Care 1995;20:25-35.

6. Amirkhan JH, Greaves H. Sense of coherence and stress: the mechanics of the healthy disposition. Psychol Health 2003;16:31-62.

7. Flannery RB, Flannery GJ. Sense of coherence, life stress, and psychological distress: A prospective methodological inquiry. J Clin Psychol 1990;46(4):415-20.

8. Bishop GD. The sense of coherence as a resource in dealing with stress. Int J Psychol Orient 1993;36(4):259-65.

9. Gallagher TJ, Wagenfeld MO, Baro F. Sense of coherence, coping and caregiver role overload. Soc Sci Med 1994;39(12):1615-22.

10. Nordstrom G, Lutzen K. Acceptance of ostomy surgery: A Swedish pilot study. Scand J Caring Sci 1995;9:11-5.

11. Drory Y, Florian V, Kravetz S. Sense of coherence: Sociodemographic variables and perceived psychological and physical health. Isr J Psychol 1991;2(2):119-25.

12. Bowman BJ. Cross-cultural validation of Antonovsky's Sense of Coherence. J Clin Psychol 1996;52(5):547-9.

13. Sagy S, Antonovsky A, Adler I. Explaining life satisfaction in later life: The sense of coherence model and activity theory. Behav Health Aging 1990;1:11-25.

14. Golińska L. Sense of coherence and satisfaction with life at its various stages. Nowiny Psychol 2003;4:33-46 [in Polish].

15. Ruiselova Z, Prokopcakova A. Sense of coherence (SOC) and its relations to cognitive (sense of control) and dynamic variables. Stud Psychol 1993;35:373-6.

16. Langius A, Bjorvell H, Antonovsky A. The sense of coherence concept and its relations to personality traits in Swedish samples. Scand J Caring Sci 1992;6(3):165-71.
17. Kalimo R, Vuori J. Work factors and health: The predictive role of pre-employment experiences. J Occup Psychol 1991;64: 97-115.

18. Johnson M. Approaching the salutogenesis of sense of coherence: the role of "active" self-esteem and coping. Brit J Health Psychol 2004;9:419-32.

19. Hanse JJ, Engstrom T. Sense of coherence and ill health among the unemployed and re-employed after closure of an assembly plant. Work Stress 1999;13(3):204-22.

20. Soderfeldt M, Soderfeldt B, Ohlson C, Theorell T, Jones I. The impact of sense of coherence and high-demand/low-control job environment on self-reported health, burnout and psychophysiological stress indicators. Work Stress 2000;14:1-15.

21. Basińska MA, Andruszkiewicz A. The sense of coherence as a predictor of healthy functioning in workplace. The study of social workers. In: Wrona-Polańska H, editor. Health, stress and illness. Kraków: Impuls; 2008. p. 63-74 [in Polish].

22. Harri M. The sense of coherence among nurse educators in Finland. Nurse Educ Today 1998;18(3):202-12.

23. Andruszkiewicz A. Sense of coherence of nurses and the pattern of behaviours connected with work [dissertation]. Bydgoszcz: Ludwik Rydygier Collegium Medicum in Bydgoszcz; 2003 [in Polish].

24. Koniarek J, Myślińska M, Nowacki H, Szymczak M. Psychosocial predictors of burnout among nurses employed in hospitals in Łódź [unpublished research report]. Łódź: Nofer Institute of Occupational Medicine; 1992 [in Polish].

25. Rogińska T, Gaida W. Strategy of coping with a mental burden in workplace. Zielona Góra: WSP; 2001 [in Polish].

26. Maslash C. Burnout: a multidimensional perspective. In: Schaufeli WB, Maslasch C, Marek T, editors. Professional burnout: recent developments in theory and research. Washington DC: Taylor \& Francis; 1993. p. 19-32.

27. Ogińska-Bulik N. Influence of coping with perceived stress in workplace on a level of burnout in a group of policemen. Przegl Psychol 2003;1:111-8 [in Polish].

28. Duffin C. Lack of breaks leaves nurses burnt out: many nurses experience loss of libido, poor sleep and constant exhaustion. Nurs Stand 2005;20(6):1-16. 
29. Borritz M, Rugulies R, Christensen KB, Villadsen E, Kristensen TS. Burnout as a predictor of self-reported sickness absence among human service workers: prospective findings from three year follow up of the PUMA study. Occup Environ Med 2006;63:98-106.

30. Pines AM. Burnout: an existential perspective. In: Schaufeli WB, Maslasch C, Marek T, editors. Professional burnout: recent developments in theory and research. Washington DC: Taylor \& Francis; 1993. p. 33-51.

31. Schaarschmidt U, Fischer AW. AVEM - pattern of behaviours connected with work. Frankfurt: Swet \& Zeitlinger; 1996 [in German].

32. Voltmer E, Kieschke U, Spahn C. Work-related behaviour and experience patterns of physicians compared to other professions. Swiss Medical Wkly 2007;137:448-53.
33. Willem A, Buelens M, De Jonghe I. Impact of organizational structure on nurses' job satisfaction. A questionnaire survey. Int J Nurs Stud 2007;44:1011-20.

34. Hobfoll SE. Stress, culture and community. Psychology and philosophy of stress. Gdańsk: Gdańskie Wydawnictwo Psychologiczne; 2006 [in Polish].

35. Hsu H-C, Kung Y-W, Huang H-C, Ho P-Y, Lin Y-Y, Chen W-S. Work stress among nursing home care attendants in Taiwan: A questionnaire survey. Int J Nurs Stud 2007;44: 736-46.

36. Villarruel AM, Bishop TL, Simpson EM, Jemmott LS, Fawcett J. Borrowed theories, shared theories, and the advancement of nursing knowledge. Nurs Sci Q 2001;14(2):158-63.

This work is available in Open Access model and licensed under a Creative Commons Attribution-NonCommercial 3.0 Poland License - http://creativecommons.org/ licenses/by-nc/3.0/pl/deed.en. 